\title{
Sobrevivência, viabilidade e controle de Alternaria sp. em sementes de feijão
}

\author{
Pâmella Soldatelli ${ }^{1}$, Ricardo Trezzi Casa ${ }^{1}$, Amanda de Lima ${ }^{1}$, Yasmin Caroline Klöckner Ribeiro ${ }^{1}$, Flávio Chupel Martins ${ }^{1}$
}

${ }^{1}$ Universidade do Estado de Santa Catarina (UDESC), Centro de Ciência Agroveterinárias (CAV), Av. Luiz de camões, 2090, Conta dinheiro, 88520-000, Lages, SC, Brasil.

Autor para correspondência: Pâmella Soldatelli (pam_soldatelli@hotmail.com)

Data de chegada: 03/07/2016. Aceito para publicação em: 17/04/2017.

$10.1590 / 0100-5405 / 2215$

\section{RESUMO}

Soldatelli, P.; Casa, R.T.; Lima, A.; Ribeiro, Y.C.K.; Martins, F.C. Sobrevivência, viabilidade e controle de Alternaria sp. em sementes de feijão. Summa Phytopathologica, v.43, n.3, p.193-198, 2017.

O objetivo deste trabalho foi avaliar a sobrevivência, a viabilidade e o controle do fungo Alternaria sp. em sementes de feijão carioca das cultivares BRS Estilo (lotes 07, 12, 201, 202, 203), BRS Tangará (lotes 01, 02, 37, 46) e preto da cultivar IPR Tuiuiu (lotes 10 e 11), IPR Uirapuru (lotes 07, 08, 12) totalizando 14 amostras. A cada 45 dias desde maio de 2014 até o final do beneficiamento (novembro de 2014), duzentas sementes por amostra foram desinfestadas e submetidas ao teste de sanidade de sementes em meio de cultura de batata-dextrose-ágar + antibiótico. Sementes de feijão colhidas na safra de 2015 no município de Muitos Capões, das cultivares BRS Estilo e IPR Tuiuiu foram submetidas a teste com fungicidas para o controle de
Alternaria sp. O teste foi realizado tratando as sementes com fungicida e semeando-as em meio de cultura batata-dextrose-ágar + antibiótico, com 200 sementes por tratamento. Os dados de incidência de Alternaria sp. em função do tempo de armazenamento foram submetidos a análise de regressão. O fungo foi detectado em todas as cultivares, lotes e épocas de amostragem. Houve redução média na incidência de Alternaria sp. das cultivares em função do tempo de $48,4 \%$ para $16,7 \%$. Ao termino do período de armazenamento o fungo manteve $34,5 \%$ de viabilidade na média das cultivares. Fungicidas com o princípio ativo fluazinam, carboxina e fludioxonil proporcionaram maior controle de Alternaria sp.

Palavras-chave: fungo, Phaseolus vulgaris, sanidade de sementes, armazenamento, tratamento de sementes.

\section{ABSTRACT}

Soldatelli, P.; Casa, R.T.; Lima, A.; Ribeiro, Y.C.K.; Martins, F.C. Survival, viability and control of Alternaria sp. in common bean seeds. Summa Phytopathologica, v.43, n.3, p.193-198, 2017.

The aim of this study was to evaluate survival, viability and control of the fungus Alternaria sp. in common bean seeds of cultivars BRS Estilo (lots 07, 12, 201, 202, 203), BRS Tangará (lots 01, 02, 37, 46) and in black bean seeds of cultivars IPR Tuiuiu (lots 10 and 11), IPR Uirapuru (lots $07,08,12$ ), totaling 14 samples. At every 45 days from May 2014 to the end of storage (November 2014), two hundred seeds per sample were disinfected and subjected to seed health test on potato-dextrose-agar culture medium + antibiotics. Bean seeds of cultivars BRS Estilo and IPR Tuiuiu, harvested in the 2015 crop season at Muitos Capões Municipality, were subjected to the test with fungicides for Alternaria sp. control. The test was carried out by treating the seeds with fungicides and sowing them on potato-dextrose-agar culture medium + antibiotics, with 200 seeds per treatment. Data of Alternaria sp. incidence as a function of storage time were subjected to regression analysis. The fungus was detected in all cultivars, lots and samplings. There was a mean reduction in the incidence of Alternaria sp. cultivars as a function of time from $48.4 \%$ to $16.7 \%$. At the end of the storage period, the fungus kept $34.5 \%$ mean viability for the average of cultivars. Fungicides with the active principle fluazinam, carboxin and fludioxonil provided greater control of Alternaria sp.

Keywords: fungus, Phaseolus vulgaris, seed health, storage, seed treatment.

Cultura de ampla importância social e econômica, o feijão (Phaseolus vulgaris L.) é cultivado em todo o território nacional e compõe um dos principais alimentos da dieta dos brasileiros.

No Brasil dois tipos de feijão são cultivados em maior escala, o grupo preto e o carioca, entretanto a preferência pelo tipo de grão para consumo e comercialização é distinta entre as regiões. O grupo preto é mais apreciado nos estados do Rio Grande do Sul, Santa Catarina e Paraná, já o grão carioca é aceito em todas as regiões do país (7).

Na safra de 2014/15 a região sul do país obteve uma produção de 954 mil toneladas de feijão, liderado pelo estado do Paraná com 724 mil toneladas, Santa Catarina com 139 mil toneladas e Rio Grande do Sul com 90 mil toneladas (6).

A produtividade da cultura é influenciada pela intensidade de doenças que ocorrem na planta em diferentes estádios do seu desenvolvimento $(3,20)$.
Muitos patógenos utilizam as sementes como mecanismo de sobrevivência por longos períodos de tempo e como agente de disseminação (24). No armazenamento os fungos podem sobreviver e manter sua viabilidade até que as sementes sejam utilizadas para semeadura. A viabilidade de fungos fitopatogênicos depende principalmente da espécie vegetal, do local de sobrevivência do fungo na semente, da incidência inicial do fungo, de condições de umidade da semente e do local de armazenamento $(2,14)$.

Os gêneros Sclerotinia, Colletotrichum, Phomopsis, Rhizoctonia e Pseudocercospora são considerados fungos patogênicos do feijão e são detectados em testes de sanidades de sementes. Estes fungos infectam as sementes podendo causar danos quando há condições ambientais favoráveis (24). Demais fungos, como Macrophomina phaseolina Tass (Goid), espécies de Fusarium e de Alternaria também são detectados nos testes de sanidade (1). 
O fungo do gênero Alternaria tem sido detectado em sementes de feijão e em sementes de diversas culturas, como o trigo (5), a soja (9), o arroz (12), o coentro (17), o algodoeiro (19) e o azevém (25).

Espécies de Alternaria são encontradas em lesões foliares de feijão (10). Há relatos da possível transmissão do fungo na semente para a planta, porém muitas vezes não são explorados do ponto de vista de entendimento desta associação (16). Os possíveis danos, a sobrevivência, a viabilidade e o controle de Alternaria em sementes de feijão não foram encontrados na bibliografia pesquisada.

O uso de sementes sadias e o tratamento de sementes com fungicidas são estratégias que podem ser empregadas, visando à redução ou a eliminação de fungos associados às sementes de feijão (11) Para a escolha do fungicida deve-se considerar a incidência do fungo nas sementes e a sensibilidade da espécie do fungo ao fungicida (22).

Os objetivos deste trabalho foram avaliar a sobrevivência do fungo Alternaria sp. em sementes de feijão carioca e preto armazenadas durante o período de entressafra em duas regiões frias do sul do país, quantificar a viabilidade do fungo ao final do armazenamento e o controle via tratamento de sementes.

\section{MATERIAL E MÉTODOS}

Os experimentos foram conduzidos no Laboratório de Fitopatologia do Centro de Ciências Agroveterinárias da Universidade do Estado de Santa Catarina, CAV, Lages, SC.

Experimento I: Sobrevivência e viabilidade de Alternaria sp. em sementes feijão.

A sobrevivência do fungo Alternaria sp. foi realizada durante o período de entressafra em sementes de feijão carioca e preto armazenadas na Cooperativa Copercampos, em Campos Novos, Santa Catarina, e na empresa NBN sementes, em Muitos Capões, Rio Grande do Sul.

Foram analisados duas cultivares de sementes de feijão carioca, BRS Estilo (lotes 7, 12) e IPR Tangará (lotes 37, 46) oriundas do município de Campos Novos, SC. Duas cultivares de sementes de feijão carioca BRS Estilo (lotes 201, 202, 203) e IPR Tangará (Lotes 01, 02) e duas cultivares de feijão preto IPR Tuiuiu (lotes 10,11) e IPR Uirapuru (lotes 07, 08, 12) oriundas do município de Muitos Capões, RS.

Em ambos os locais, as sementes de feijão sem tratamento químico permaneceram armazenadas em sacos de polipropileno, suportada por paletes, em armazéns de alvenaria com umidade relativa, temperatura e ventilação ambiente.

As amostras enviadas ao laboratório foram coletadas por responsáveis das empresas identificando-se as cultivares e os lotes seguindo a exigência das Regras de Análises de Sementes (4).

Foram feitas cinco avaliações num intervalo de 45 dias entre cada avaliação, perfazendo um período de tempo de 225 dias, o que corresponde ao período de entressafra da cultura na região sul do Brasil. A primeira avaliação foi realizada no mês de maio e a última no mês de novembro de 2014.

As sementes de cada amostra foram desinfestadas com solução de hipoclorito de sódio (1\%) durante dois minutos, com posterior enxágue, com água destilada e estéril. Após, as sementes foram predispostas em papel filtro esterilizado para secagem e posteriormente semeadas em meio de cultura BDA+A (Batata-Dextrose-Ágar + antibiótico $=200$ $\mathrm{mg} \mathrm{L}^{-1}$ de sulfato de estreptomicina) (8).

Para cada cultivar foram analisadas 200 sementes por lote, com 25 sementes distribuídas em caixas de acrílico do tipo gerbox em oito repetições. O material foi incubado em câmara de crescimento à temperatura de $25^{\circ} \mathrm{C}$ e fotoperíodo de $12 \mathrm{~h}$, durante sete dias. $\mathrm{O}$ delineamento experimental foi completamente casualizado.

Foi considerada infectada a semente sobre o qual foi possível identificar a colônia ou estruturas dos fungos sob lupa estereoscópica em aumento de 40 vezes. A confirmação da presença dos fungos foi feita com montagem de lâmina em microscópio ótico, analisando-se as estruturas fúngicas e comparando com as descritas na literatura $(10,26)$.

Os dados da incidência de Alternaria sp. em sementes foram submetidos à análise de variância (1\%). Os resultados da relação entre incidência de Alternaria sp. e tempo de armazenamento foram submetidos à análise de regressão. A viabilidade do fungo foi calculada utilizando-se regra de três simples, segundo a fórmula:

$$
\mathrm{V}(\%)=\left[\frac{1 \times 100}{\mathrm{I}}\right]-100
$$

Considerando que $\mathrm{V}$ é a viabilidade do fungo ao final do período de armazenamento, i é a incidência inicial e I é a incidência final do fungo em cada cultivar e lote.

Experimento II: Controle de Alternaria sp. com fungicidas via tratamento de sementes.

O tratamento de sementes foi feito com fungicidas e doses descritos na tabela. O critério de escolha dos fungicidas foi baseado em produtos registrados para a cultura do feijão e para fungos em geral nas sementes.

Utilizaram-se sementes de feijão carioca BRS Estilo e de feijão preto IPR Tuiuiu colhidas na safra 2015 no município de Muitos Capões, RS. Os produtos foram dosados com auxílio de uma pipeta de precisão e misturados com água até completar um volume de calda de $0,7 \%$ do peso da amostra (700 g). A mistura das sementes com o fungicida foi feita em saco plástico, via úmida, agitando-o manualmente até obter cobertura uniforme das sementes. Após três dias do tratamento, as sementes foram semeadas em caixas gerbox contendo meio de cultura de BDA + A, conforme descrito no Ensaio I.

O delineamento experimental foi inteiramente casualizado. Para cada tratamento foram analisadas 200 sementes, com 25 sementes por gerbox em oito repetições. As sementes foram incubadas em câmara de crescimento em temperatura de $25^{\circ} \mathrm{C}$ e fotoperíodo de $12 \mathrm{~h}$, durante sete dias.

Nas avaliações foi quantificado o número de sementes colonizadas pelo fungo Alternaria sp. Foi considerada infectada a semente sobre o qual foi possível identificar a colônia ou estruturas do fungo sob lupa estereoscópica em aumento de 40 vezes.

Os dados obtidos foram submetidos à análise de variância e as médias comparadas pelo teste de Tukey (5\%). Os resultados foram expressos em incidência do fungo e percentagem de controle.

\section{RESULTADOS E DISCUSSÃO}

Experimento I: Sobrevivência e viabilidade de Alternaria sp. em sementes feijão.

O fungo Alternaria sp. foi detectado nas quatro cultivares de feijão em todas as amostras durante o período de entessafra, com significativa redução na detecção à medida que aumentou o período de armazenamento. $\mathrm{O}$ fungo manteve a viabilidade nas sementes de feijão ao final do armazenamento.

Nas sementes das cultivares oriundas do município de Campos Novos as reduções do início ao final do armazenamento foram de $18,0 \%$ para $6,5 \%$ em BRS Estilo/07, de $15,0 \%$ para $4,5 \%$ em BRS 
Tabela 1. Fungicidas e doses utilizados no tratamento de sementes de feijão nas cultivares BRS Estilo e IPR Tuiuiu. Lages, SC. 2015.

\begin{tabular}{|c|c|c|c|c|}
\hline \multirow[t]{2}{*}{ Tratamento } & \multirow[t]{2}{*}{ Igrediente ativo } & \multirow[t]{2}{*}{ Produto comercial } & \multicolumn{2}{|c|}{ Dose (100 Kg de sementes) } \\
\hline & & & g/i.a. & mL/p.c. \\
\hline 2. & Carbendazim+tiram & Derosal Plus & $45+105$ & 300 \\
\hline 3. & Carbendazim & Derosal & 50 & 100 \\
\hline 4. & Carboxina + tiram & Vitavax & $60+60$ & 300 \\
\hline 5. & Difenoconazol & Spectro & 5 & 33,4 \\
\hline 7. & Fluquinconazol & Atento & 50 & 300 \\
\hline 8. & Metalaxil+Tiabendazol+Fludioxonil & Maxim Advanced & $20+150+25$ & 125 \\
\hline 9. & Metalaxil+Fludioxonil+Carbendazim+ Tiram & Maxim XL+Derosal Plus & $10+25+45+105$ & $300+300$ \\
\hline 10. & Metalaxil+Fludioxonil & Maxim XL & $10+25$ & 300 \\
\hline
\end{tabular}

Estilo/12, de 20,0\% para 3,5\% em IPR Tangará/37 e 14,5\% para 3,0\% em IPR Tangará/46.

Nas sementes das cultivares oriundas do município de Muitos Capões, no grupo carioca verificaram-se reduções de $32,5 \%$ para $13,5 \%$ em BRS Estilo/201, 41,5\% para 19,0\% em BRS Estilo/202, 33,5\% para 8,5\% em BRS Estilo/203, 27,0\% para 7,0\% em IPR Tangará/01 e $32,5 \%$ para $12,0 \%$ em IPR Tangará/02. No grupo preto obtiveram-se reduções de $25,5 \%$ para $10,5 \%$ em IPR Tuiuiu/ $10,26,5 \%$ para $8,0 \%$ em IPR Tuiuiu/11, 33,0\% para 13,5\% em IPR Uirapuru/07, 32,0\% para $15,0 \%$ em IPR Uirapuru/08 e 31,5\% para 11,0\% em IPR Uirapuru/ 12 .

Resultados semelhantes foram encontrados em sementes de triticale com redução na incidência de Alternaria Alternata (Fr.FR.) Keissler, de $35 \%$ para $5 \%$ após doze meses de armazenamento (15). Em sementes de coentro, também houve a redução na incidência do fungo $A$. alternata de $95 \%$ para $31 \%$ após doze meses de armazenamento (17).

Considerando $100 \%$ de viabilidade de Alternaria sp. na primeira avaliação, ao final do armazenamento observou-se redução de $33,8 \%$ na média de todos os lotes e cultivares. Ao analisar as sementes oriundas de Campos Novos, na média das cultivares, Alternaria sp. apresentou $26,0 \%$ de viabilidade no final do armazenamento. Nas sementes de Muitos Capões, RS, Alternaria sp. obteve $37,0 \%$ de viabilidade no final do armazenamento. Observa-se que a maior incidência inicial foi nas cultivares e lotes de sementes de Muitos Capões, o que pode ter propiciado a maior viabilidade final de Alternaria sp.

Os resultados mostram que Alternaria sp. sobrevive infectando sementes de feijão desde o beneficiamento das sementes (maio) até o momento da semeadura (dezembro) nas regiões frias do sul do país. Resultados similares foram obtidos para $A$. alternata em sementes de trigo armazenadas durante nove meses e o fungo manteve viabilidade de $50,0 \%$ ao final do armazenamento (13). Em sementes de triticale armazenadas durante doze meses $A$. alternata manteve viabilidade de $12,0 \%$ ao final do armazenamento (15) Já em sementes de coentro A. alternata manteve $32,6 \%$ de viabilidade após seis meses de armazenamento (17).

Normalmente análises de sanidade de sementes são efetuadas próximas da data de semeadura, o que corresponde no sul do Brasil
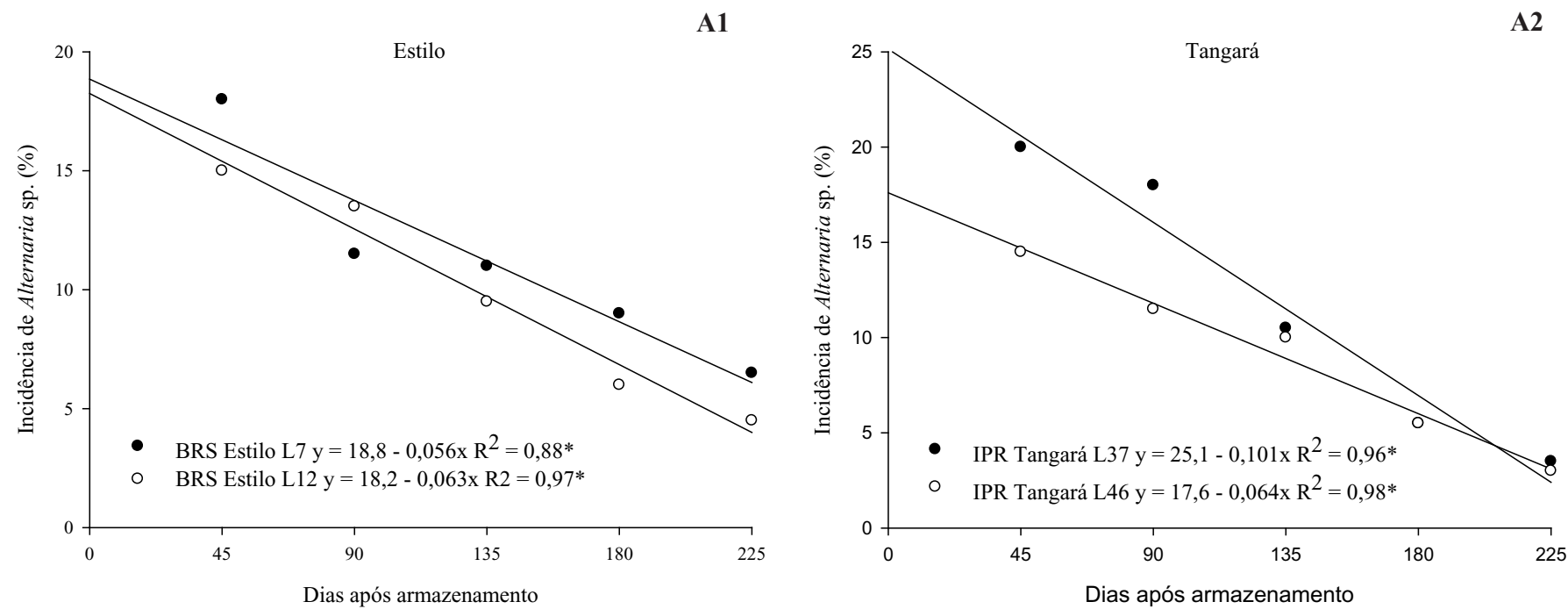

Figura 1. Relação entre incidência de Alternaria sp. (\%) e tempo de armazenamento (dias) para lotes de sementes de feijão das cultivares BRS Estilo (A1) e IPR Tangará (A2) do município de Campos Novos, SC. *Significativo o nível de 1\% de probabilidade pelo teste de Tukey. 

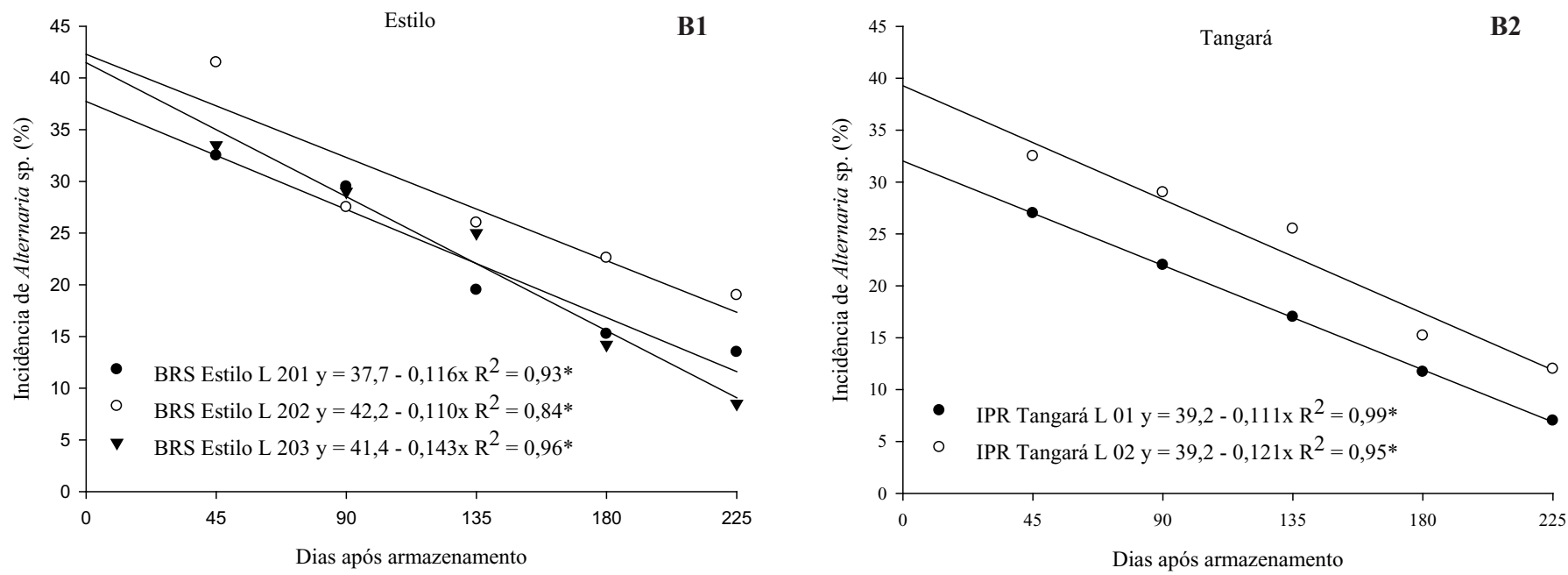

Figura 2. Relação entre incidência de Alternaria sp. (\%) e tempo de armazenamento (dias), para lotes de sementes de feijão das cultivares BRS Estilo (B1) e IPR Tangará (B2) do município de Muitos Capões, RS. *Significativo o nível de 1\% de probabilidade pelo teste de Tukey.

Tabela 2. Incidência e viabilidade de Alternaria sp. em sementes de feijão carioca e preto durante o armazenamento em Unidades de Beneficiamento de Sementes em Campos Novos e em Muitos Capões. Lages, SC. 2014.

\begin{tabular}{|c|c|c|c|c|}
\hline \multirow{6}{*}{ Campos Novos } & Cultivar/lote & Inc. Inicial & Inc. Final & $\begin{array}{l}\text { Viabilidade } \\
\text { final }\end{array}$ \\
\hline & Estilo/7 & 18 & 6,5 & 36 \\
\hline & Estilo/12 & 15 & 4,5 & 30 \\
\hline & Tangará/37 & 20 & 3,5 & 17,5 \\
\hline & Tangará/46 & 14,5 & 3 & 20,5 \\
\hline & Média & 16,9 & 4,4 & 26 \\
\hline \multirow{12}{*}{ Muitos Capões } & Estilo/201 & 32,5 & 13,5 & 41,5 \\
\hline & Estilo/202 & 41,5 & 19 & 46 \\
\hline & Estilo/203 & 33,5 & 8,5 & 25 \\
\hline & Tangrá/01 & 27 & 7 & 26 \\
\hline & Tangrá/02 & 32,5 & 12 & 37 \\
\hline & Tuiuiu/10 & 25,5 & 10,5 & 41 \\
\hline & Tuiuiu/11 & 26,5 & 8 & 30 \\
\hline & Uirapuru/07 & 33 & 13,5 & 41 \\
\hline & Uirapuru/08 & 32 & 15 & 47 \\
\hline & Uirapuru/12 & 31,5 & 11 & 35 \\
\hline & Média & 31,6 & 11,8 & 37 \\
\hline & Média geral & 27,3 & 9,6 & 33,8 \\
\hline
\end{tabular}
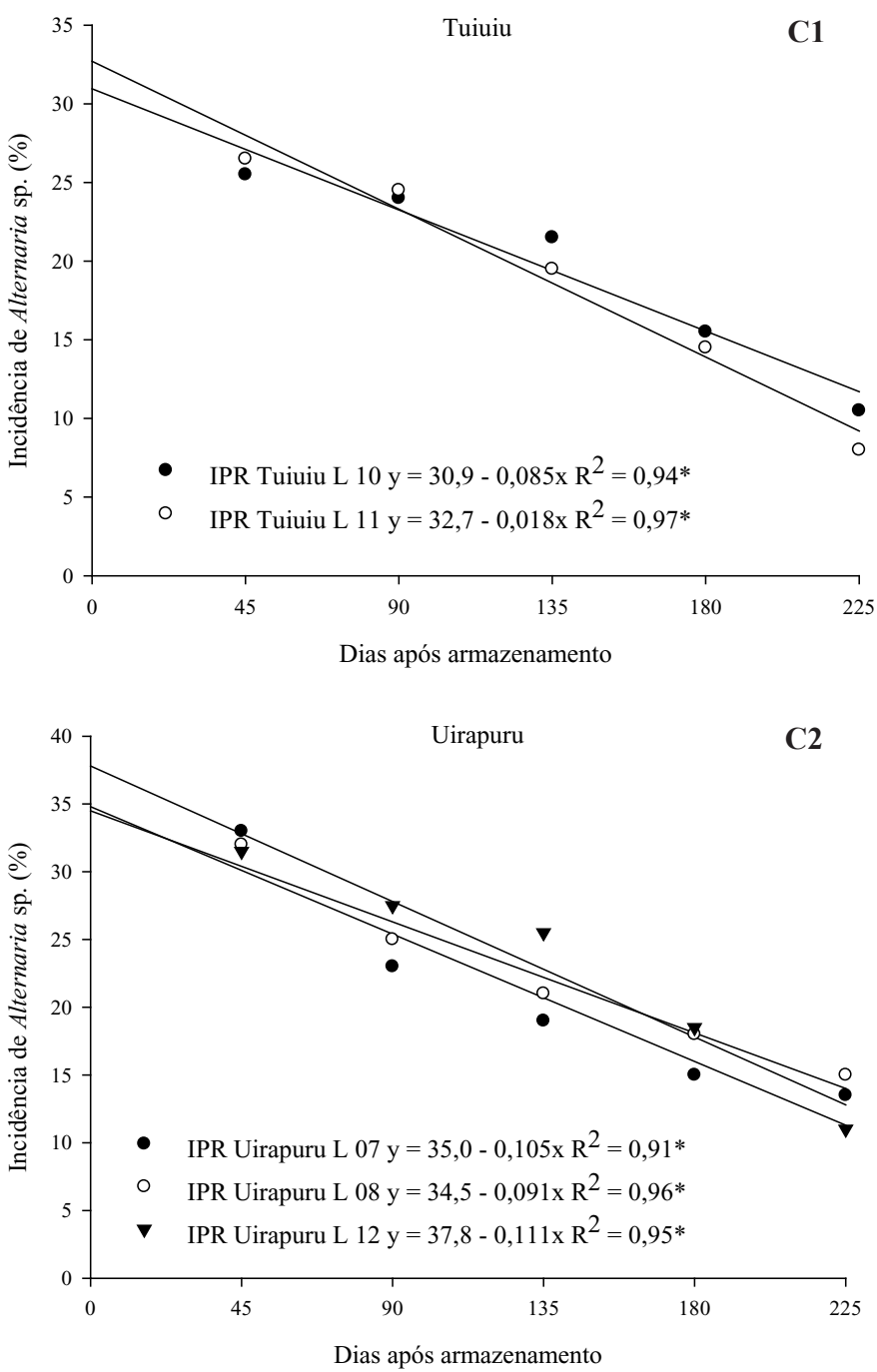

Figura 3. Relação entre incidência de Alternaria sp. (\%) e tempo de armazenamento (dias), para lotes de sementes de feijão das cultivares IPR Tuiuiu (C1) e IPR Uirapuru (C2) do município de Muitos Capões, RS. *Significativo o nível de $1 \%$ de probabilidade pelo teste de Tukey. 
Tabela 3. Efeito do tratamento de sementes com diferentes fungicidas na incidência e no controle de Alternaria sp. em sementes de feijão BRS Estilo e IPR Tuiuiu, semeadas em meio de cultura Batata-Dextrose-Ágar. Lages, SC. 2015.

\begin{tabular}{|c|c|c|c|c|c|c|}
\hline & \multicolumn{4}{|c|}{ Incidência } & \multicolumn{2}{|c|}{ Controle } \\
\hline \multirow[t]{2}{*}{ Tratamentos } & \multicolumn{2}{|c|}{ BRS Estilo } & \multicolumn{2}{|c|}{ IPR Tuiuiu } & BRS Estilo & IPR Tuiuiu \\
\hline & \multicolumn{6}{|c|}{--_- } \\
\hline 2. Carbendazim+tiram & 25,4 & de & 17,5 & $\mathrm{~cd}$ & 12,8 & 25,5 \\
\hline 3. Carbendazim & 63,0 & $\mathrm{a}$ & 40,0 & a & 0,0 & 0,0 \\
\hline 6. Fluazinam & 0,5 & hi & 0,5 & $f$ & 97,7 & 97,8 \\
\hline 7. Fluquinconazol & 43,0 & $\mathrm{bc}$ & 36,5 & $\mathrm{ab}$ & 0,0 & 0,0 \\
\hline 8. Metalaxil+tiabendazol+fludioxonil & 1,5 & hi & 1,0 & $f$ & 93,3 & 95,7 \\
\hline 9. Metalaxil+fludioxonil+carbendazim+tiram & 0,0 & $\mathrm{i}$ & 0,0 & $\mathrm{f}$ & 100 & 100 \\
\hline 13. Tiofanato metílico & 44 & $\mathrm{bc}$ & 39,5 & $\mathrm{a}$ & 0,0 & 0,0 \\
\hline C.V. $(\%)$ & 17,6 & & 22,0 & & & \\
\hline
\end{tabular}

um tempo aproximado de seis a oito meses de armazenamento. Considerando este fato espera-se uma redução na incidência de Alternaria sp., porém o fungo ainda pode se manter viável. Desta forma o tratamento de sementes com fungicidas específicos é recomendável.

Experimento II: Controle químico de Alternaria sp. com fungicida em sementes de feijão.

Alguns fungicidas reduziram significativamente a incidência do fungo Alternaria sp. nas sementes de feijão, com destaque para: carboxim + tiram, fluazinam, metalaxil + tiabendazol + fludioxonil, metalaxil + fludioxonil + carbendazim + tiram, metalaxil + fludioxonil e tiofanato metílico + fluazinam. O controle obtido por estes fungicidas foi respectivamente de $91,1 \%, 97,7 \%, 93,3 \%, 100,0 \%, 95,5$ e $95,5 \%$ na cultivar BRS Estilo e 85,1\%, 97,8\%, 95,7, 100,0\%, 100,0\% e 100,0\% na cultivar IPR Tuiuiu.

O bom desempenho destes fungicidas provavelmente + deve-se aos ingredientes ativos fluazinam, carboxina e fludioxonil, uma vez que o gênero Alternaria é insensível aos fungicidas benzimidazóis (carbendazin, tiabendazol, tiofanato metílico) (23). Já o fungicida fenilamida (metalaxil) tem ação fungitóxica sobre os gêneros Pythium e Phytophtora e não sobre Alternaria (18).

O presente estudo demonstrou que as sementes de feijão carioca (BRS Estilo e IPR Tangará) e preto (IPR Tuiuiu e IPR Uirapuru) mantém a sobrevivência e a viabilidade de Alternaria sp. e que o período de entressafra em regiões frias no sul do Brasil. O período em que as sementes permanecem armazenadas não é suficiente para eliminar o inóculo deste fungo. Da mesma forma, demonstrou que o fungo pode ser controlado pelo tratamento de sementes com os ingredientes ativos fluazinam, carboxina e fludioxonil.

\section{REFERÊNCIAS}

1. Alves, M.C.; Pozza, E.A.; Machado, J.C.; Carvalho, M.G.G. Desenvolvimento e validação de um sistema especialista para identificar fungos na análise sanitária de sementes. Revista Brasileira de Sementes, Campinas, v.28, n.1, p.176-186, 2006

2. Agarwal, V.K.; Sinclair, J.B. Principles of seed pathology. 2. ed. Boca Raton: Lewis Publishers, 1997. 539p.

3. Bianchini, A.; Maringoni, A.C.; Carneiro, S.M.T.P.G. Doenças do feijoeiro. In: Kimati, H.; Amorim, L.; Bergamin Filho, A.; Camargo, L.E.A.; Rezende, J.A.M. Manual de fitopatologia: doenças das plantas cultivadas. 3.ed. São Paulo: Agronômica Ceres, 1997. v.1, p.353-381.

4. Brasil. Ministério da Agricultura Pecuária e Abastecimento. Teste de Sanidade de Sementes. In: REGRAS para a análise de sementes. Brasília, DF: MAPA/ACS, 2009. cap. 9, p.335-340.

5. Brasil. Companhia Nacional de Abastecimento. Acompanhamento de safra brasileira: grãos. In: Décimo primeiro levantamento, agosto 2015. Brasília, 2015. v.2, n.11, p.1-101. Disponível em: $<$ http://www.conab.gov. br/OlalaCMS/uploads/arquivos/16_06_09_09_00_00_boletim_graos_junho_2016__final.pdf >. Acesso em: 15 mai. 2016.

6. Casa, R.T.; Kuhnem, P.R.; Bogo, A.; Belani, A.M.M.; Bolzan, J.M.; Oliveira, F.S.; Blum, M.M.C. Survey, survival and control of Alternaria alternata in wheat seeds. Revista Brasileira de Sementes, Campinas, v.34, n.3 p.358-365, 2012.

7. Comissão técnica sul-brasileira de feijão - CTSBF. Informações técnicas para o cultivo de feijão na Região Sul brasileira. 2.ed. Florianópolis: Epagri, 2012.157p.

8. Fernandez, M.R. Manual para laboratório de fitopatologia. Passo Fundo: Embrapa-CNTP, 1993. 128p.

9. Goulart, A.C.P. Fungos em sementes de soja: detecção, importância e controle. Dourados: Embrapa Agropecuária Oeste, 2004. 72p.

10. Hall, R. Compendium of bean diseases. St. Paul: American Phytopathological Society, 1991. 73p.

11. Kimati, H. Controle químico. In: Amorim. L.; Rezende, J.A.M.; Bergamin, A.F. Manual de fitopatologia: princípios e conceitos. 4.ed. São Paulo: Agronômica Ceres, 2011. v.1, p.343-365.

12. Macedo, E.C.; Groth, D.; Soave, J. Influência da embalagem e do armazenamento na qualidade sanitária de sementes de arroz. Revista Brasileira de Sementes, Campinas, v.24, n.1, p.42-50, 2002.

13. Malaker, P.K.; Mian, I.H.; Bhuiyan, A.M.; Akanda, A.M.; Reza, M.M.A. Effect of storage containers and time on seed quality of wheat. Bangladesh journal of Agricultural Research, Gazipur, v.33, n.3, p.469-477, 2008.

14. Maude, R.D. Seedborne diseases an their control: principles and practice. Wallingford: CAB International, 1996. 280p.

15. Medina, F.P.; Tanaka, M.A.S.; Parisi, J.J.D., Sobrevivência de fungos associados ao potencial fisiológico de sementes de triticale (X. triticosecale 
Wittmack) durante o armazenamento. Revista Brasileira de Sementes, Campinas, v.31, n.4, p.17-26, 2009.

16. Moraes, M.H.D.; Menten, J.O.M. Transmissão de Alternaria spp. através de sementes de feijão e seu efeito sobre a qualidade fisiológica das sementes. Summa Phytopathologica, Botucatu, v.32, n.4, p.381-383, 2006.

17. Nascimento, W.M.; Pereira, R.S.; Freitas, R.A.; Blumer, L.; Muniz, M.F.B. Colheita e armazenamento de sementes de coentro. Pesquisa Agropecuária Brasileira, Brasília, DF, v.41, n.12, p.1793-1801, 2006.

18. Pinto, N.F.J.A. Tratamento de sementes de milho com fungicidas. Revista Ceres, Viçosa, v.50, n.291, p.681-686, 2003.

19. Pizzinatto, M.A.; Cia, E.; Parisi, J.J.D.; Medina, P.F.; Fuzatto, M.G. Associação de Alternaria macrospora e A. alternata a sementes de algodoeiro e sua ação patogênica. Summa Phytopathologica, Botucatu, v.31, n.4, p.311-318, 2005 .

20. Reis, A.; Satelis, J.F.; Pereira, R.S.; Nascimento, W.M. Associação de Alternaria dauci e A. alternata com sementes de coentro e eficiência do tratamento químico. Horticultura Brasileira, Brasília, DF, v.24, n.1, p.107-111, 2006.

21. Reis, E.M.; Casa, R.T. Doenças dos cereais de inverno: diagnose, epidemiologia e controle. 2.ed. Lages, 2007. 176p.

22. Reis, E.M.; Reis, A.C.; Carmona, M.A. Fungitoxicidade e sensibilidade. In: Reis, E.M.; Reis, A.C.; Carmona, M.A. Manual de fungicidas. Passo Fundo: UPF, 2010. cap.5, p.26-29.

23. Sartorato, A.; Rava, C.A. Patologia de sementes. In: Vieira, E.H.N.; Rava, C.A. Sementes de feijão: produção e tecnologia. Santo Antônio de Goiás: Embrapa Arroz e Feijão, 2000. cap.9, p.201-215.

24. Silva, A.E.L.; Reis, E.M.; Tonin, R.F.B.; Danelli, A.L.D.; Avozani, A. Identificação e quantificação de fungos associados a sementes de azevém (Lolium multiflorum Lam.). Summa Phytopathologica, Botucatu, v.40, n.2, p.156-162, 2014

25. Simmons, E.G. Alternaria: an identification manual. Utrecht: CBS Fungal Biodiversity Centre, 2007. 775p. 